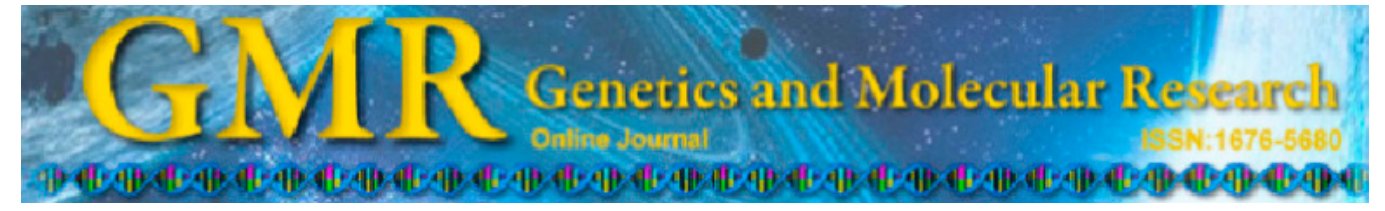

\title{
Impact of methylenetetrahydrofolate reductase polymorphisms and folate intake on the risk of gastric cancer and their association with Helicobacter pylori infection and tumor site
}

J. Chen', L. Yuan', Y.Q. Duan', J.Q. Jiang ${ }^{3}$, R. Zhang', Z.J. Huang' ${ }^{1}$ and X.R. Xiao ${ }^{1}$

${ }^{1}$ Department of Cadre Ward, The General Hospital of Chengdu Military Area, Chengdu, China ${ }^{2}$ Department of Nuclear Medicine, The General Hospital of Chengdu Military Area, Chengdu, China ${ }^{3}$ Department of Thoracic Surgery,

The General Hospital of Chengdu Military Area, Chengdu, China

Corresponding author: X.R. Xiao

E-mail:sun_suxia@yahoo.com.cn

Genet. Mol. Res. 13 (4): 9718-9726 (2014)

Received December 12, 2012

Accepted October 18, 2013

Published January 24, 2014

DOI http://dx.doi.org/10.4238/2014.January.24.2

\begin{abstract}
Folic acid and methylenetetrahydrofolate reductase (MTHFR) may both affect the development of human cancer. We conducted a population-based case-control study in a Chinese population to investigate the potential role of folate intake and MTHFR gene polymorphisms in gastric cancer, and their interaction with infection by Helicobacter pylori and tumor location. A total of 767 patients with newly diagnosed gastric cancer and 775 controls were selected for this study. Genotyping of MTHFR C677T and A1298C was conducted by TaqMan assays using the ABI Prism 7911HT Sequence Detection System, and information
\end{abstract}


on folate intake was collected by questionnaire. Compared with the $\mathrm{CC}$ genotype of MTHFR C677T, the TT genotype was significantly associated with a decreased risk of gastric cancer when the analysis was adjusted for other potential risk factors. We found a marginal significantly decreased risk of gastric cancer for individuals carrying the $\mathrm{T}$ allele [adjusted odds ratio $(\mathrm{OR})=0.83 ; 95 \%$ confidence interval $(\mathrm{CI})=0.65-1.01]$. We detected an inverse relationship between folate intake and risk of gastric cancer, and the adjusted ORs $(95 \% \mathrm{CI})$ for moderate and high folate intake were 0.97 (0.74-1.25) and 0.64 (0.49-0.87), respectively. Moreover, H. pylori infection, folate intake, and location of the tumor showed a significant interaction with the MTHFR C677T polymorphism. Our study suggests a protective role of MTHFR 677TT and high folate intake against gastric cancer, and the effect of the MTHFR C677T genotype may differ by $H$. pylori infection, folate consumption, and tumor site.

Key words: Methylenetetrahydrofolate reductase; Polymorphisms; Folate intake; Gastric cancer

\section{INTRODUCTION}

Although gastric cancer incidence and mortality have been decreasing around the world, it is still the most common cause of cancer death in China for both genders (IARC, 2008). Although Helicobacter pylori strains have been proposed to be a major cause of gastric cancer, infections by H. pylori do not account completely for the incidence of gastric cancer. Epidemiological studies have indicated an association between folate intake and a decreased risk of certain cancers (Larsson et al., 2006; Yoo et al., 2012; de Cássia Carvalho et al., 2012; Promthet et al., 2012; Ding et al., 2012), including gastric cancer (Yoo et al., 2012), esophageal cancer (Larsson et al., 2006) and ovarian cancer (Ding et al., 2012). Folate is a key B vitamin required for 1-carbon metabolism and is critical for DNA methylation, synthesis, and repair (Jemal et al., 2007). Deficiency of folate has been suggested to increase the risk of colorectal cancer because folate deficiency results in irregular DNA methylation and imbalance in DNA precursors (Eichholzer et al., 2001; La Vecchia et al., 2002).

Methylenetetrahydrofolate reductase (MTHFR) is an enzyme that converts 5,10-methylenetetrahydrofolate to 5-methyltetrahydrofolate, the prevalent form of circulating folate and the methyl donor for the conversion of homocysteine to methionine. Two common MTHFR gene polymorphisms, MTHFR C677T (rs1801133) and A1298C (rs1801131), have been studied extensively. The MTHF TT and CC variants result in low-activity MTHFR enzymes (Frosst et al., 1995; van der Put et al., 1998) resulting in low levels of circulating 5-methyltetrahydrofolate. The MTHFR 677TT genotype has been associated with a lower cancer risk compared with the homozygous CC genotype (Hubner and Houlston, 2007).

We have previously reported that folate intake and MTHFR 677CT/TT are associated with an increased risk of esophageal cancer, and that folate shows a significant interaction with the MTHFR C677T polymorphism (APJCP). The association of MTHFR with susceptibility to gastric cancer has been investigated by several recent studies, whose results, however, were inconclusive (Dong et al., 2010). Several studies have reported that the homozygous genotype of the 
MTHFR C677T polymorphism is associated with an increased risk of gastric cancer (Graziano et al., 2006; Lacasana-Navarro et al., 2006), whereas other studies have reported that this polymorphism is associated with a decreased gastric cancer risk (Mu et al., 2004; Kim et al., 2005; Weng et al., 2006). However, these studies did not take into account possible interactions between MTHFR gene polymorphisms and folate intake, H. pylori infection, or tumor site. Therefore, we conducted a large-scale case-control study in a Chinese population to evaluate the potential role of folate intake and MTHFR gene polymorphisms in gastric cancer, and to investigate possible interactions of folate intake, $H$. pylori infection, and tumor sites with MTHFR polymorphisms.

\section{MATERIAL AND METHODS}

\section{Study population}

The study population comprised 767 patients with newly diagnosed gastric cancer and 775 population-based controls. All enrolled patients were confirmed for their pathological condition by the General Hospital of Chengdu Military Area between March 2008 and May 2011. Subjects with secondary or recurrent tumors were excluded. The tumor stages were classified according to the TNM classification, including clinical or pathological TNM stages. Gastric cancer was classified by anatomical site (cardia or non-cardia) and histological types (intestinal, diffuse, or mixed type).

The control group consisted of participants in the health examination center from April 2007 and April 2008, and they were matched with cancer patients by age and gender. All patients were asked to provide their peripheral blood, and they had read and signed an informed consent form.

Our study was approved by the ethics committee of the General Hospital of Chengdu Military Area in China.

\section{Data collection}

A self-administered structured questionnaire, consisting of 65 questions, was used in this study. Information collected included demographic data (age, gender, and family history of cancer) and clinical characteristics (histopathology, tumor location, and lymph modes status), tobacco use, smoking, and alcohol-consumption and dietary habits (including 45 foods/food groups). Cigarette smoking was measured in pack-years [number of cigarettes smoked per day/20 x smoking time (in years)] and divided into 2 categories: smokers who consumed less than 40 packs/year, and $\geq 40$ packs/year or more. Alcohol consumption was calculated from the amount of alcohol consumed per day in grams. The subjects were classified into two categories: drinkers who consumed less than $22.8 \mathrm{~g}$ alcohol per day, and those consuming $\geq 22.8$ alcohol per day.

For each food item, we collected the frequency and quantity of consumption, and calculated the daily intake by multiplying the frequency reported for the consumption of each food item by the specified portion size. The folate intake was computed by multiplying the food intake (in g) and the folate content (per g) of food in our questionnaire, and then the sum of the combined folate intake from various foods/food groups was calculated as the total folate intake. Trained interviewers conducted face-to-face interviews with all subjects to obtain the above information. Completed questionnaires were obtained from 767 cases and 775 controls. Cancer patients were asked to refer about habits a year before the disease diagnosed. 


\section{Diagnosis of $H$. pylori infection}

Infection by H. pylori was diagnosed by enzyme-linked immunosorbent assay (ELISA) with IgG antibodies (HpIgG ELISA) using a commercially available kit (Genesis Diagnostics, Cambridgeshire, UK) according to manufacturer instructions on sera obtained from 5 $\mathrm{mL}$ of blood. The sensitivity and specificity of the kit was 91 and $100 \%$, respectively.

\section{Blood samples and DNA collection}

All participants provided $5 \mathrm{~mL}$ of blood, which was stored at $-20^{\circ} \mathrm{C}$. Genomic DNA was extracted from whole-blood samples using the Qiagen Blood Kit (Qiagen, Chatsworth, CA, USA). Genotyping was conducted by TaqMan assays using the ABI Prism 7911HT Sequence Detection System (Applied Biosystems, Foster City, CA). Primer, probes, and reaction conditions were those described in our previous study (Jing et al., 2012). Briefly, a total PCR reaction volume of $10 \mathrm{uL}$ contained $200 \mathrm{ng}$ of genomic DNA and 20 pmol of each primer. The PCR conditions were as follows: initial denaturation at $94^{\circ} \mathrm{C}$ for $5 \mathrm{~min}$, followed by 35 cycles at $94^{\circ} \mathrm{C}$ for $65 \mathrm{~s}, 60^{\circ} \mathrm{C}$ for $65 \mathrm{~s}$, and $72^{\circ} \mathrm{C}$ for $90 \mathrm{~s}$; a final extension was performed at $72^{\circ} \mathrm{C}$ for $5 \mathrm{~min}$. After brief centrifugation, agarose gel electrophoresis was conducted. The PCR products included a 173-bp fragment of 677C/C wild-type homozygotes; 173-, 125-, and 48-bp fragments of 677C/T heterozygotes; and 125- and 48-bp fragments of $677 \mathrm{~T} / \mathrm{T}$ homozygotes. The genotyping was performed by laboratory personnel blinded to the case-control status. For quality-control purposes, we also genotyped internal positive control samples, used no-template controls, and replicated the genotyping of $10 \%$ of the samples.

\section{Statistical analysis}

The differences between the patients and controls were estimated by logistic regression analysis. Adjusted odds ratios (ORs) were calculated with a logistic regression model that controlled for gender and age and are indicated with their $95 \%$ confidence intervals (CIs). Subjects with the wild-type genotypes were considered the baseline group. The expected frequency of control genotypes was checked by the Hardy-Weinberg equilibrium test. Unconditional logistic regression was undertaken to estimate ORs and their $95 \% \mathrm{CIs}$ after controlling for potentially confounding factors.

Folate intake was computed by multiplying food intake ( $\mu \mathrm{g} /$ day) by folate content $(\mu \mathrm{g} /$ day) of the food; the sum of the folate intake from all foods was calculated to obtain the total intake. Folate intake was categorized as low, moderate, or high using tertiles as cut-off points. We assessed the role of folate intake in the association between MTHFR and gastric cancer by subgroup analysis of folate intake levels. All analyses were performed by using the the SPSS version 16.0 statistical software (SPSS, Chicago, IL, USA). The descriptive data for the major characteristics of study groups are expressed as means and percentages.

\section{RESULTS}

The general characteristics of the study population are shown in Table 1 . The mean age of gastric cancer patients was $62.4 \pm 12.3$ years and that of controls $62.7 \pm 11.8$ years. Drink- 
ers and smokers were at higher risk for gastric cancer than non-drinkers and non-smokers $(\mathrm{P}$ $<0.05)$. Patients with $H$. pylori infection were also at higher risk for gastric cancer $(\mathrm{P}<0.05)$.

\begin{tabular}{|c|c|c|c|c|c|}
\hline Characteristics & $\begin{array}{c}\text { Cases } \\
\mathrm{N}=767\end{array}$ & $\%$ & $\begin{array}{l}\text { Controls } \\
\mathrm{N}=775\end{array}$ & $\%$ & $\mathrm{P}$ value \\
\hline Age (years) & $62.4 \pm 12.3$ & & $62.7 \pm 11.8$ & & \\
\hline$<60$ & 270 & 35.2 & 283 & 36.5 & \\
\hline $60-70$ & 322 & 42 & 316 & 40.8 & \\
\hline$>70$ & 175 & 22.8 & 176 & 22.7 & 0.85 \\
\hline \multicolumn{6}{|l|}{ Gender } \\
\hline Male & 478 & 62.3 & 480 & 61.9 & \\
\hline Female & 289 & 37.7 & 295 & 38.1 & 0.88 \\
\hline \multicolumn{6}{|c|}{ Smoking habit (package/year) } \\
\hline$<40$ & 481 & 62.7 & 531 & 68.5 & \\
\hline$\geq 40$ & 286 & 37.3 & 244 & 31.5 & 0.016 \\
\hline \multicolumn{6}{|c|}{ Drinking habit (g/day) } \\
\hline$<22.8^{\circ}$ & 503 & 65.6 & 564 & 72.8 & \\
\hline$\geq 22.8$ & 264 & 34.4 & 211 & 27.2 & 0.002 \\
\hline \multicolumn{6}{|c|}{ H. pylori infection } \\
\hline Positive & 549 & 71.6 & 392 & 50.6 & \\
\hline Negative & 218 & 28.4 & 383 & 49.4 & $<0.001$ \\
\hline \multicolumn{6}{|l|}{ TNM stage } \\
\hline I & 266 & 34.7 & & & \\
\hline II & 208 & 27.1 & & & \\
\hline III & 139 & 18.1 & & & \\
\hline IV & 154 & 20.1 & & & \\
\hline \multicolumn{6}{|l|}{ Tumor site } \\
\hline Cardiac & 167 & 21.8 & & & \\
\hline Non-cardiac & 600 & 78.2 & & & \\
\hline \multicolumn{6}{|c|}{ Histological type } \\
\hline Intestinal & 401 & 52.3 & & & \\
\hline Diffuse & 297 & 38.7 & & & \\
\hline Mixed & 69 & 9 & & & \\
\hline
\end{tabular}

The distributions for the MTHFR C677T and A1298C genotypes and their adjusted ORs and 95\%CIs in gastric cancer are shown in Table 2. The distributions of the MTHFR C677T and A1298C gene polymorphisms in the controls were in Hardy-Weinberg equilibrium. The frequencies of the MTHFR C677T CC, CT, and TT genotypes were 50.9, 37.7, and $11.4 \%$ among gastric cancer patients and $46.7,39.7$, and $13.6 \%$ among controls, respectively. The frequencies of the MTHFR A1298C AA, AC, and CC genotypes were 41.1, 46.9, and 12.0 among gastric cancer patients and $42.7,46.2$, and $11.1 \%$ among controls, respectively. For MTHFR C677T, the TT genotype was significantly associated with a decreased risk of gastric cancer when compared with the $\mathrm{CC}$ genotype and adjusted for potential risk factors. We found a marginal significantly decreased risk of gastric cancer for individuals with the T allele (adjusted OR, 0.83; 95\% CI, 0.65-1.01). In contrast, none of the MTHFR A1298C polymorphisms was significantly associated with increased risk of gastric cancer.

The mean folate intakes in cancer patients and controls were $261.3 \pm 24.7 \mu \mathrm{g} / \mathrm{day}$ and $295.5 \pm 29.6 \mu \mathrm{g} /$ day, respectively. We detected an inverse relationship between folate intake and risk of gastric cancer, and the adjusted OR and 95\%CI for folate intakes of 230-310 $\mu \mathrm{g} /$ day and $>310 \mu \mathrm{g} /$ day were $0.97(0.74-1.25)$ and 0.64 (0.49-0.87), respectively.

The effects of interactions of the MTHFR C677T polymorphisms with smoking, drinking, and $H$. pylori infection on the relative risk of developing gastric cancer is shown in 
Table 3. When we used the MTHFR 677CC genotype as the reference, smoking and drinking did not modify the association between the MTHFR C677T polymorphism and the risk of gastric cancer. In contrast, $H$. pylori infection and folate intake each showed a significant interaction with the MTHFR C677T polymorphism: individuals who were not infected by $H$. pylori had a lower risk of developing gastric cancer when they had the MTHFR 677TT genotype (P for interaction $<0.05$ ). Moreover, higher folate intake was associated with a decreased risk of gastric cancer in individuals with the MTHFR 677CT and TT genotypes.

\begin{tabular}{|c|c|c|c|c|c|c|}
\hline MTHFR & $\begin{array}{c}\text { Cases } \\
\mathrm{N}=767\end{array}$ & $\%$ & $\begin{array}{l}\text { Controls } \\
\mathrm{N}=775\end{array}$ & $\%$ & OR & Adjusted $\mathrm{OR}^{1}$ \\
\hline \multicolumn{7}{|l|}{ C677T } \\
\hline $\mathrm{CC}$ & 390 & 50.9 & 362 & 46.7 & 1.0 (Ref.) & 1.0 (Ref.) \\
\hline $\mathrm{CT}$ & 289 & 37.7 & 307 & 39.7 & $0.87(0.70-1.09)$ & $0.84(0.67-1.05)$ \\
\hline TT & 88 & 11.4 & 105 & 13.6 & $0.78(0.56-1.08)$ & $0.71(0.51-0.97)$ \\
\hline $\mathrm{T}$ allele & 377 & 49.1 & 412 & 53.3 & $0.85(0.69-1.04)$ & $0.83(0.65-1.01)$ \\
\hline \multicolumn{7}{|l|}{ A1298C } \\
\hline AA & 316 & 41.1 & 331 & 42.7 & 1.0 (Ref.) & 1.0 (Ref.) \\
\hline $\mathrm{AC}$ & 359 & 46.9 & 358 & 46.2 & $1.05(0.84-1.31)$ & $1.09(0.88-1.52)$ \\
\hline $\mathrm{CC}$ & 92 & 12.0 & 86 & 11.1 & $1.12(0.79-1.58)$ & $1.40(0.86-1.67)$ \\
\hline $\mathrm{C}$ allele & 451 & 58.9 & 444 & 57.3 & $1.06(0.86-1.31)$ & $1.13(0.91-1.46)$ \\
\hline \multicolumn{7}{|c|}{ Folate intake ( $\mu \mathrm{g} /$ day $)$} \\
\hline Means (SE) & $261.3 \pm 24.7$ & & $295.5 \pm 29.6$ & & & \\
\hline$<230$ & 335 & 43.7 & 300 & 38.7 & 1.0 (Ref.) & 1.0 (Ref.) \\
\hline $230-310$ & 296 & 38.6 & 280 & 36.1 & $0.96(0.75-1.20)$ & $0.97(0.74-1.25)$ \\
\hline$>310$ & 136 & 17.7 & 195 & 25.2 & $0.62(0.47-0.82)$ & $0.64(0.49-0.87)$ \\
\hline
\end{tabular}

${ }^{1}$ Adjusted for age, gender, smoking, drinking, and H. pylori infection.

Table 3. Interaction between MTHFR C677T and MTHFR A1298C polymorphisms and smoking, drinking and H. pylori infection for gastric cancer risk.

\begin{tabular}{|c|c|c|c|}
\hline Characteristics & CT vs CC OR $(95 \% \mathrm{CI})^{1}$ & TT vs CC OR $(95 \% \mathrm{CI})^{1}$ & $\mathrm{P}$ for interaction \\
\hline \multicolumn{4}{|c|}{ Smoking habit (package/year) } \\
\hline$<40$ & $0.81(0.52-1.08)$ & $0.59(0.33-0.86)$ & \\
\hline$\geq 40$ & $0.91(0.62-1.21)$ & $0.80(0.52-1.02)$ & 0.07 \\
\hline \multicolumn{4}{|c|}{ Drinking habit (g/day) } \\
\hline$<22.8$ & $0.82(0.61-1.06)$ & $0.68(0.47-0.95)$ & \\
\hline$\geq 22.8$ & $0.88(0.66-1.09)$ & $0.73(0.47-0.98)$ & 0.38 \\
\hline \multicolumn{4}{|c|}{ H. pylori infection } \\
\hline Positive & $0.91(0.68-1.13)$ & $0.78(0.53-1.04)$ & \\
\hline Negative & $0.75(0.58-0.96)$ & $0.63(0.43-0.86)$ & 0.012 \\
\hline \multicolumn{4}{|l|}{ Folate intake } \\
\hline$<230$ & $0.96(0.71-1.23)$ & $0.85(0.52-1.23)$ & \\
\hline $230-300$ & $0.81(0.57-0.96)$ & $0.75(0.53-1.04)$ & \\
\hline$>310$ & $0.74(0.45-0.87)$ & $0.59(0.41-0.86)$ & 0.006 \\
\hline
\end{tabular}

${ }^{1}$ Adjusted for age and gender.

The association between MTHFR C677T polymorphism and different locations of the gastric cancer tumors in the gastric system is shown in Table 4. The MTHFR C677T polymorphism was not associated with an increased risk of cardia cancer, whereas the MTHFR 677TT genotype was associated with a decreased risk of non-cardia cancer. 
Table 4. MTHFR C677T and MTHFR A1298C genotype distributions and adjusted OR for different tumor sites.

\begin{tabular}{|c|c|c|c|c|c|c|c|c|}
\hline & \multirow[t]{2}{*}{ Controls } & \multirow[t]{2}{*}{$\%$} & \multicolumn{3}{|c|}{ Cardiac cancer } & \multicolumn{3}{|c|}{ Non-cardiac cancer } \\
\hline & & & Cases $\mathrm{N}=167$ & $\%$ & OR $(95 \% \mathrm{CI})^{1}$ & Cases N $=600$ & $\%$ & OR $(95 \% \mathrm{CI})^{1}$ \\
\hline \multicolumn{9}{|l|}{ C677T } \\
\hline $\mathrm{CC}$ & 362 & 46.7 & 77 & 46.0 & 1.0 (Ref.) & 313 & 52.2 & 1.0 (Ref.) \\
\hline CT & 307 & 39.7 & 66 & 39.4 & $1.01(0.69-1.48)$ & 223 & 37.2 & $0.84(0.66-1.06)$ \\
\hline TT & 105 & 13.6 & 24 & 14.6 & $1.07(0.62-1.82)$ & 64 & 10.6 & $0.44(0.27-0.68)$ \\
\hline
\end{tabular}

${ }^{1}$ Adjusted for age, gender, smoking, drinking, and H. pylori infection.

\section{DISCUSSION}

This study evaluated the association of the MTHFR C677T and MTHFR A1298C gene polymorphisms with increased susceptibility to gastric cancer. The MTHFR C677T polymorphism decreased the risk of gastric cancer, as did high folate consumption whose protective effect was modified by polymorphisms in the MTHFR C677T gene. Moreover, we observed that a MTHFR C677T polymorphism significantly interacted with $H$. pylori infection and folate intake in the development of gastric cancer.

Previous studies on the MTHFR C677T and MTHFR A1298C polymorphisms and their associations with gastric cancer risk have yielded inconsistent results. Studies conducted in China (Qin et al., 2008), Iran (Saberi et al., 2012), Korea (Cui et al., 2010), Mexico (ZunigaNoriega et al., 2007; Galvan-Portillo et al., 2009), Norway (Vollset et al., 2007), and Denmark (Zacho et al., 2011) identified a significant association of the MTHFR C677T polymorphism with gastric cancer. Studies in China, Iran, and Denmark indicated a significantly increased risk of gastric cancer, with ORs ranging from 1.4-2.6 (Qin et al., 2008; Zacho et al., 2011; Saberi et al., 2012). In contrast, several other studies have indicated that MTHFR 677TT significantly protects against gastric cancer (Galvan-Portillo et al., 2009; Cui et al., 2010). Cui and colleagues, studying a Korean population, reported that the MTHFR 677T allele has a significant protective effect against gastric cancer, indicated by ORs of CT versus CC of 0.81 (95\%CI, 0.69-0.94) (Cui et al., 2010). In a Mexican population, a significant reduction in gastric cancer risk was observed among individuals with high folate intake and who carried the MTHFR 677TT genotype (Galvan-Portillo et al., 2009). Two other studies detected a non-significant protective effect on gastric cancer risk (Zuniga-Noriega et al., 2007; Vollset et al., 2007). Here, we observed that MTHFR 677TT was associated with a reduced risk of gastric cancer, and the MTHFR 677 T allele was associated with a marginally decreased risk. Moreover, the MTHFR 677TT genotype is associated with a protective effect against various cancers, including breast and colorectal cancers (Chou et al., 2006; Cui et al., 2010). The observed differences in the effects of the MTHFR C677T polymorphisms on gastric cancer risk in the different studies might be due to population background, sample sizes, or environmental factors and other factors. Further confirmation of the differential effects observed here is strongly needed.

Folate mediates the transfer of 1-carbon moieties in the synthesis of nucleotides required for DNA synthesis, replication, and repair, and also in DNA methylation reactions, and these processes may play a critical role in carcinogenesis (Wang et al., 2008; Mason, 2009). An abundant intake of folate-rich foodstuffs was more likely to convey protection against developing some cancers (Mason, 2009). Our study indicating a protective function of folate intake against increased gastric cancer risk has also been observed in previous epidemiological 
studies (Chou et al., 2006; Vollset et al., 2007; Cui et al., 2010). Moreover, the results of these studies suggest that a higher intake of folate could greatly reduce the risk of cancer among individuals with the MTHFR 677TT genotype.

H. pylori infection is an important etiological factor in gastric cancer, and previous studies have indicated that $H$. pylori infection affects the induction of methylation in human gastric mucosa (Chan et al., 2003; Maekita et al., 2006; Tahara et al., 2009), and suggested that aberrant DNA methylation is one of the major events occurring early in tumorigenesis. The results of our study identified a significant interaction between $H$. pylori infection and MTHFR C677T polymorphisms on the effect on gastric cancer risk, which indicated that $H$. pylori infection of the gastric mucosa may lead to transcriptional inactivation of specific genes and may increase DNA damage, mutation, or chromosomal instability.

In summary, this case-control study has investigated the association between folate intake and the MTHFR C677T and MTHFR A1298C variant genotypes in the development of gastric cancer. Its results suggest a protective role of MTHFR 677TT and high folate intake against gastric cancer, and the effect of the MTHFR C677T genotype may differ by $H$. pylori infection, folate consumption, and different tumor sites. Gastric cancer appears to be the result of an interaction between genes and the environment, and further studies with larger sample sizes are needed to confirm any environmental and genetic associations in this disease.

\section{REFERENCES}

Chan AO, Lam SK, Wong BC, Wong WM, et al. (2003). Promoter methylation of E-cadherin gene in gastric mucosa associated with Helicobacter pylori infection and in gastric cancer. Gut 52: 502-506.

Chou YC, Wu MH, Yu JC, Lee MS, et al. (2006). Genetic polymorphisms of the methylenetetrahydrofolate reductase gene, plasma folate levels and breast cancer susceptibility: a case-control study in Taiwan. Carcinogenesis 27: 22952300.

Cui LH, Shin MH, Kweon SS, Kim HN, et al. (2010). Methylenetetrahydrofolate reductase C677T polymorphism in patients with gastric and colorectal cancer in a Korean population. BMC Cancer 10: 236.

de Cássia Carvalho BR, da Costa DM, Cordeiro DE, Vieira AP, et al. (2012). Interaction of MTHFR C677T and A1298C, and MTR A2756G gene polymorphisms in breast cancer risk in a population in Northeast Brazil. Anticancer Res. 32: 4805-4811.

Ding XP, Feng L and Ma L (2012). MTHFR C677T polymorphism and ovarian cancer risk: a meta-analysis. Asian Pac. J. Cancer Prev. 13: 3937-3942.

Dong X, Wu J, Liang P, Li J, et al. (2010). Methylenetetrahydrofolate reductase C677T and A1298C polymorphisms and gastric cancer: a meta-analysis. Arch. Med. Res. 41: 125-133.

Eichholzer M, Luthy J, Moser U and Fowler B (2001). Folate and the risk of colorectal, breast and cervix cancer: the epidemiological evidence. Swiss Med. Wkly. 131: 539-549.

Frosst P, Blom HJ, Milos R, Goyette P, et al. (1995). A candidate genetic risk factor for vascular disease: a common mutation in methylenetetrahydrofolate reductase. Nat. Genet. 10: 111-113.

Galvan-Portillo MV, Cantoral A, Onate-Ocana LF, Chen J, et al. (2009). Gastric cancer in relation to the intake of nutrients involved in one-carbon metabolism among MTHFR 677 TT carriers. Eur. J. Nutr. 48: 269-276.

Graziano F, Kawakami K, Ruzzo A, Watanabe G, et al. (2006). Methylenetetrahydrofolate reductase 677C/T gene polymorphism, gastric cancer susceptibility and genomic DNA hypomethylation in an at-risk Italian population. Int. J. Cancer 118: 628-632.

Hubner RA and Houlston RS (2007). MTHFR C677T and colorectal cancer risk: A meta-analysis of 25 populations. Int. J. Cancer120: 1027-1035.

International Agency for Research on Cancer (2008). Globocan 2008: Stomach Cancer incidence, Mortality and Prevalence Worldwide in 2008. IARC.

Jemal A, Siegel R, Ward E, Murray T, et al. (2007). Cancer statistics, 2007. CA Cancer J. Clin. 57: 43-66.

Jing C, Huang Z, Duan Y, Xiao X, et al. (2012). Folate intake, methylenetetrahydrofolate reductase polymorphisms in association with the prognosis of esophageal squamous cell carcinoma. Asian Pac. J. Cancer Prev. 13: 647-651. 
Kim JK, Kim S, Han JH, Kim HJ, et al. (2005). Polymorphisms of 5,10-methylenetetrahydrofolate reductase and risk of stomach cancer in a Korean population. Anticancer Res. 25: 2249-2252.

La Vecchia C, Negri E, Pelucchi C and Franceschi S (2002). Dietary folate and colorectal cancer. Int. J. Cancer 102: 545-547.

Lacasana-Navarro M, Galvan-Portillo M, Chen J, Lopez-Cervantes M, et al. (2006). Methylenetetrahydrofolate reductase $677 \mathrm{C}>\mathrm{T}$ polymorphism and gastric cancer susceptibility in Mexico. Eur. J. Cancer 42: 528-533.

Larsson SC, Giovannucci E and Wolk A (2006). Folate intake, MTHFR polymorphisms, and risk of esophageal, gastric, and pancreatic cancer: a meta-analysis. Gastroenterology 131: 1271-1283.

Maekita T, Nakazawa K, Mihara M, Nakajima T, et al. (2006). High levels of aberrant DNA methylation in Helicobacter pylori-infected gastric mucosae and its possible association with gastric cancer risk. Clin. Cancer Res. 12: 989-995.

Mason JB (2009). Folate, cancer risk, and the Greek god, Proteus: a tale of two chameleons. Nutr. Rev. 67: 206-212.

Mu LN, Ding BG, Chen CW, Wei GR, et al. (2004). A case-control study on the relationship between methyl-tetrahydrofolic acid reductase 677 gene polymorphism and the risk of stomach cancer. Zhonghua Liu Xing Bing Xue Za Zhi. 25: 495-498.

Promthet S, Pientong C, Ekalaksananan T, Songserm N, et al. (2012). Risk factors for rectal cancer and methylenetetrahydrofolate reductase polymorphisms in a population in Northeast Thailand. Asian Pac. J. Cancer Prev. 13: 4017-4023.

Qin JM, Wang XM, Chen B, Yang L, et al. (2008). Study on the ingestion of folate and polymorphism of MTHFR C677T with esophageal cancer in Xinjiang Kazakh. Zhonghua Liu Xing Bing Xue Za Zhi. 29: 30-33.

Saberi S, Zendehdel K, Jahangiri S, Talebkhan Y, et al. (2012). Impact of methylenetetrahydrofolate reductase C677T polymorphism on the risk of gastric cancer and its interaction with Helicobacter pylori infection. Iran Biomed. J. 16: $179-184$

Tahara T, Arisawa T, Shibata T, Nakamura M, et al. (2009). Increased number of methylated CpG islands correlates with Helicobacter pylori infection, histological and serological severity of chronic gastritis. Eur. J. Gastroenterol. Hepatol. 21: 613-619.

van der Put NM, Gabreels F, Stevens EM, Smeitink JA, et al. (1998). A second common mutation in the methylenetetrahydrofolate reductase gene: an additional risk factor for neural-tube defects? Am. J. Hum. Genet. 62: 1044-1051.

Vollset SE, Igland J, Jenab M, Fredriksen A, et al. (2007). The association of gastric cancer risk with plasma folate, cobalamin, and methylenetetrahydrofolate reductase polymorphisms in the European Prospective Investigation into Cancer and Nutrition. Canc. Epidemiol. Biomarkers Prev. 16: 2416-2424.

Wang J, Sasco AJ, Fu C, Xue H, et al. (2008). Aberrant DNA methylation of P16, MGMT, and hMLH1 genes in combination with MTHFR C677T genetic polymorphism in esophageal squamous cell carcinoma. Canc. Epidemiol. Biomarkers Prev. 17: 118-125.

Weng YR, Sun DF, Fang JY, Gu WQ, et al. (2006). Folate levels in mucosal tissue but not methylenetetrahydrofolate reductase polymorphisms are associated with gastric carcinogenesis. World J. Gastroenterol. 12: 7591-7597.

Yoo JY, Kim SY, Hwang JA, Hong SH, et al. (2012). Association study between folate pathway gene single nucleotide polymorphisms and gastric cancer in Koreans. Genomics Inform. 10: 184-193.

Zacho J, Yazdanyar S, Bojesen SE, Tybjaerg-Hansen A, et al. (2011). Hyperhomocysteinemia, methylenetetrahydrofolate reductase c. $677 \mathrm{C}>\mathrm{T}$ polymorphism and risk of cancer: cross-sectional and prospective studies and meta-analyses of 75,000 cases and 93,000 controls. Int. J. Cancer128: 644-652.

Zuniga-Noriega JR, Velazco-Campos MR, Aguirre-Rodriguez A, Villarreal LM, et al. (2007). C677T polymorphism of the MTHFR gene and the risk of developing distal gastric cancer in a Mexican population. Rev. Gastroenterol. Mex. 72: $355-358$. 